\title{
Design and Status of the Balloon Experimental Twin Telescope for Infrared Interferometry (BETTII): An Interferometer at the Edge of Space
}

\author{
S. A. Rinehart ${ }^{1, a}$, R. B. Barclay ${ }^{a}$, R. K. Barry ${ }^{a}$, D. J. Benford ${ }^{a}$, P. C. Calhoun ${ }^{a}$,D. J. Fixsen ${ }^{\mathrm{a}, \mathrm{b}}$, E. T. \\ Gorman ${ }^{\mathrm{a}}$, M. L. Jackson ${ }^{\mathrm{a}}$, C. A. Jhabvala ${ }^{\mathrm{a}}$, D. T. Leisawitz, S. F. Maher, ${ }^{\mathrm{a}, \mathrm{c}}$, J. E. Mentzella ${ }^{\mathrm{a}}$. L.G. \\ Mundy $^{\mathrm{b}}$, M. J. Rizzo ${ }^{\mathrm{a}, \mathrm{b}}$, R. F. Silverberg ${ }^{\mathrm{a}}$, J. G. Staguhn ${ }^{\mathrm{a}, \mathrm{d}}$ \\ ${ }^{a}$ NASA's Goddard Space Flight Center, Greenbelt MD 20771 \\ ${ }^{b}$ Department of Astronomy, University of Maryland, College Park, MD 20741 \\ ${ }^{\mathrm{c}}$ Science System Applications, Inc. \\ ${ }^{\mathrm{d} J o h n s ~ H o p k i n s ~ U n i v e r s i t y ~}$
}

\begin{abstract}
The Balloon Experimental Twin Telescope for Infrared Interferometry (BETTII) is an 8-meter baseline far-infrared interferometer designed to fly on a high altitude balloon. BETTII uses a double-Fourier Michelson interferometer to simultaneously obtain spatial and spectral information on science targets; the long baseline permits subarcsecond angular resolution, a capability unmatched by other far-infrared facilities. Here, we present key aspects of the overall design of the mission and provide an overview of the current status of the project. We also discuss briefly the implications of this experiment for future space-based far-infrared interferometers.
\end{abstract}

Keywords: Interferometry, Far-Infrared, Balloon

\section{INTRODUCTION}

The Balloon Experimental Twin Telescope for Infrared Interferometry (BETTII; Figure 1) is an eight-meter baseline Michelson interferometer designed to fly on a high-altitude balloon. The long baseline will provide unprecedented angular resolution $\left(\sim 0.5^{\prime \prime}\right)$ in the $30-90 \mu \mathrm{m}$ band. This band is inaccessible from the ground; the high atmospheric transmission at balloon altitudes, in combination with BETTII's unique double-Fourier instrument will allow spectral resolution of up to $\mathrm{R} \equiv \lambda / \Delta \lambda \sim 200$. The integration of these capabilities allows BETTII to provide spatially resolved spectroscopy, a new tool for exploring astronomical regimes that remain currently hidden from our view. Simultaneously, BETTII will advance key technologies and techniques to readiness for spaceborne interferometry..

Previously, we have reported upon the scientific objectives of the BETTII mission (Rinehart 2010); in short, the prime science goal of the first BETTII flight will be to study clustered star formation, with secondary scientific objectives of obtaining spatially resolved spectroscopy of the cores of Active Galactic Nuclei. In this paper we focus on the design work that is currently complete for BETTII. BETTII was selected for funding in fall 2010, and the official project start was January 3, 2011. In Section 2, we discuss mechanical aspects of the BETTII design. The optical design is covered in Section 3. Section 4 will discuss other aspects of the BETTII design. We also note that several major components of the BETTII design are being presented in other papers at this conference, notably the fringe tracker subsystem (Rizzo et al. 2012) and the control system architecture (Benford et al. 2012).

\section{MECHANICAL SYSTEMS}

In designing BETTII, one of the driving technical requirements is that pointing jitter must be tightly controlled (Table 1). Small variations in the pointing of the gondola leads to phase noise contributions in the measured interferogram; while at low frequencies, such phase noise can be eliminated through careful data reduction and calibration, high frequency oscillations can cause "blurring" of the individual fringes, leading to a reduction in the apparent fringe visibility and a

${ }^{1}$ Contact S. Rinehart at Stephen.A.Rinehart@nasa.gov ,ph: 301-286-4591

Optical and Infrared Interferometry III, edited by Françoise Delplancke, Jayadev K. Rajagopal, Fabien Malbet, Proc. of SPIE Vol. 8445, 844508 · (c) 2012 SPIE · CCC code: 0277-786/12/\$18 · doi: 10.1117/12.926376 
reduction in the overall data quality. This leads to a structural design that is strong, lightweight, and which has a high natural frequency, in order to rapidly damp out any oscillations within the system. This is critical for minimizing phase noise. In the balloon environment, fortunately, there are no external forces that excite high frequency oscillations; the most significant perturbation is the pendulation of the gondola itself, which occurs at frequencies $<1 \mathrm{~Hz}$. Since the amplitudes of these oscillations can be relatively large ( $\sim 10$ arcminutes, Fixsen et al. 1996), we require a number of different mechanisms to compensate for these effects, as well as mechanisms to allow us to obtain the interferometric fringes in the time domain (i.e. a delay line).

\subsection{Structure}

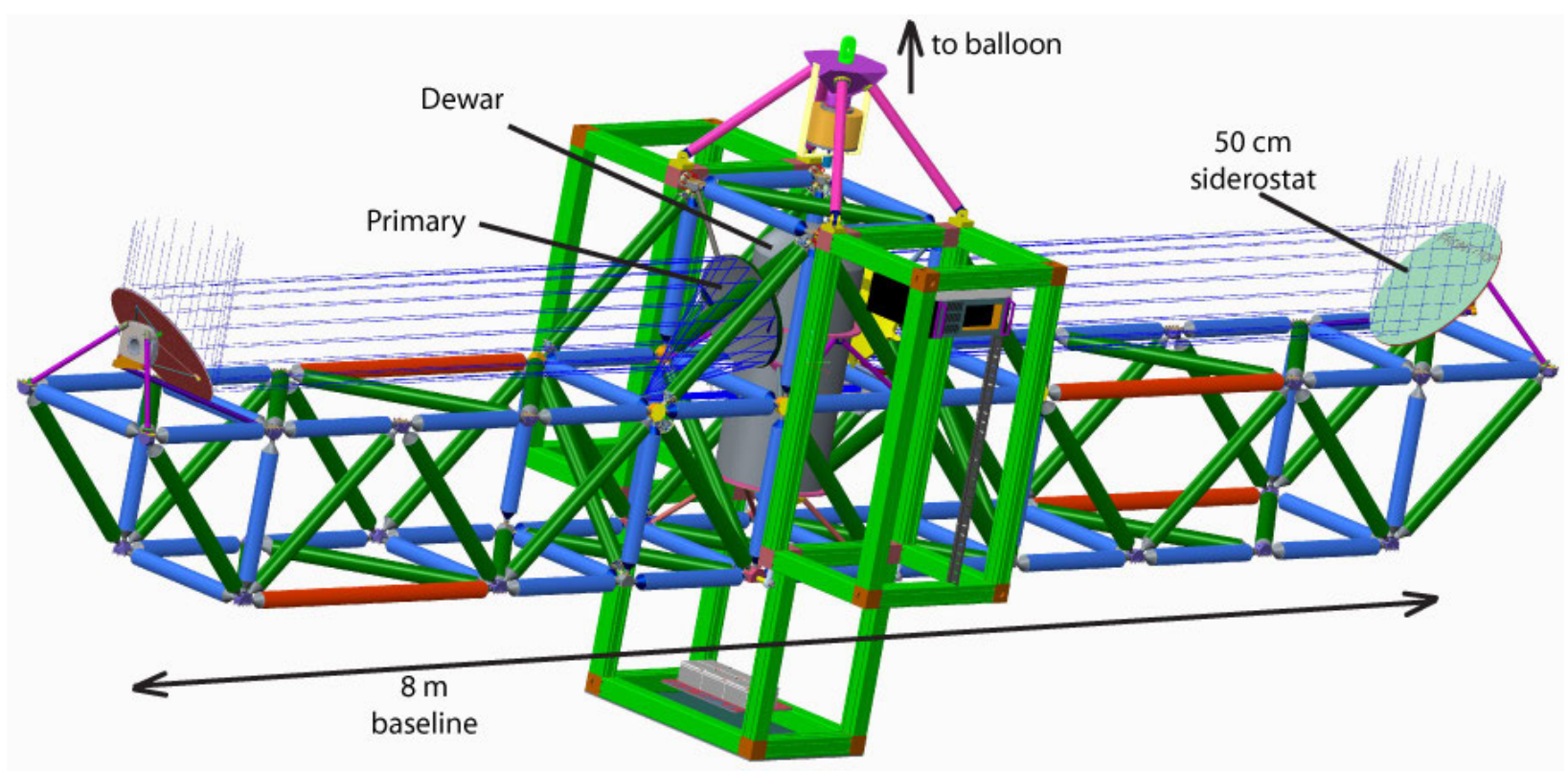

Figure 1: By using a metering truss made of carbon fiber and steel, we create a structure that is strong, stiff, and which behaves well under temperature change. Individual mounts for siderostats, the cryostat, etc. use a combination of titanium and Invar tubing to provide stiffness and to match CTE effects. Surrounding the center of the metering truss is a load structure made of 80/20 aluminum, designed to hold non-optical components of the BETTII system.

The BETTII truss structure has two major components; the first of these, and the focus of this discussion, is a carbon fiber metering truss that extends through the full length of the gondola. The second is a series of external boxes constructed from 80/20 aluminum that surround the core of the instrument, dubbed the "load frame". The carbon fiber structure is designed to hold all optical elements of BETTII, including the instrument dewar, while the aluminum outer boxes are designed for holding electronics and mechanisms needed for control of the gondola. These aluminum boxes will be have passive vibration isolation from the carbon fiber, in order to minimize any transmission of mechanism perturbations into the optical system.

The carbon fiber structure consists of 112 individual carbon fiber tubes with a 3 -inch inner diameter and a 0.06 " wall. In each end of each tube, a stainless steel "nosecone" is epoxied into place, completing the individual strut. We have already built and tested 5 of these tubes, using an Instron machine. These tests revealed that all of the tubes met strength requirements; three of the tubes failed during testing, and all of these failures occurred where the epoxy was bonded to the carbon fiber. The first failure occurred at over $30 \%$ beyond the strength requirement, while the other two both occurred more than a factor of two above the requirement. Two tubes remained intact up to the maximum force applied by the Instron machine. The struts are then assembled using stainless steel nodes (Figure 2); the entire structure is assembled in a "tinker-toy" way, with stainless steel spherical washers between the noses and nodes of the horizontal and vertical tubes, and HDPE washers in a similar position for the diagonal tubes. This structure is nearly kinematic. By using HDPE washers for the diagonal struts, we compensate for thermal effects as the structure varies in temperature from the ground $(\sim 30 \mathrm{C})$ to float altitude $(\sim-50 \mathrm{C})$. 
This design was modeled in detail using finite element analysis, and we carefully studied the first five modes of oscillation. The first mode, shown in Figure 3, is a "flapping" mode, with the two arms of the interferometer moving symmetrically side-to-side (relative to the gravity vector). The frequency of this mode is $24.8 \mathrm{~Hz}$, well above any external perturbing forces, leading to relatively fast damping of any vibrations in the gondola itself. Because it is perpendicular to the boom, the effect on pathlength is second-order. Further, because this mode is symmetric, it produces relatively little differential piston (phase) error between the two arms of the interferometer, provided that we are able to compensate for the shift in the pointing of the two telescopes. The second mode is similar; a "vertical flapping" mode, where the two ends of the interferometer move vertically with respect to the gravity vector. The natural frequency of this mode is $37.5 \mathrm{~Hz}$, and like the first mode, this produces symmetric perturbations that reduce the overall impact on fringe quality.

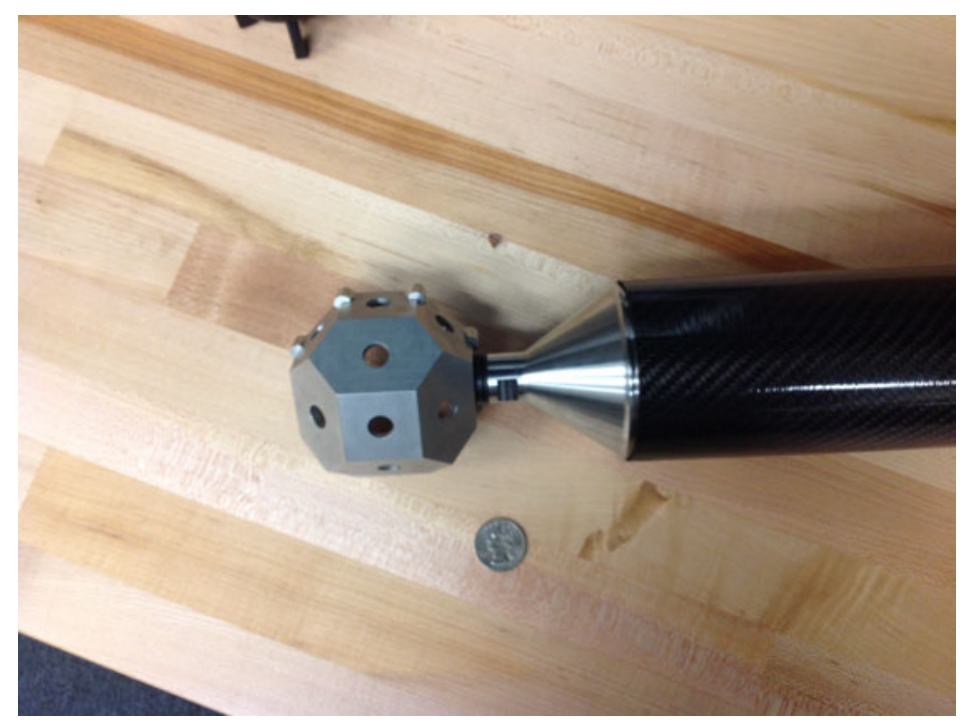

Figure 2: The metering truss consists of carbon fiber tubes with steel nosecones epoxied into each end. These tubes are then bolted into stainless steel nodes, allowing a "tinker-toy" construction of the structure.

In order to maximize the safety of the payload, and to improve the likelihood that we will be able to re-fly BETTII after our first campaign, we have also examined its behavior under different load scenarios. Of most importance is the behavior of the gondola under a 10-g vertical acceleration (this occurs for balloon payloads after the gondola has been released, when the parachute deploys). This analysis shows that the majority of the truss structure experiences only $\sim 1$ $\mathrm{Ksi}$; the highest stresses are observed where the dewar is mounted to the gondola, at $30 \mathrm{Ksi}$. These stainless steel structures are each designed to a load capacity of $80 \mathrm{Ksi}$. Additional analysis also explored the effect of a $5 \mathrm{~g}$ diagonal load on the gondola; results from that study also demonstrated that the gondola is sufficiently strong to bear this load.

Finally, and perhaps most critically, we studied the thermal behavior of the gondola. The first BETTII launch will most likely be a spring launch out of Fort Sumner, New Mexico, but it is conceivable that delays could instead lead to a summer launch in Palestine, Texas. In these ground environments, temperatures can be as high as $\sim 35 \mathrm{C}$, while the ambient temperature at float altitude is $\sim-50 \mathrm{C}$ (the ambient temperature, as the balloon rises through the atmosphere, can actually be significantly lower than $-50 \mathrm{C}$, but because of the rapid ascent, the payload is not expected to thermalize to these lower temperatures). The $\sim 80$ degree temperature variation from launch to float has the potential to lead to deformation of the structure, which can in turn lead to misalignment of optics. For this reason, the entire structure is designed to deform homologously with temperature. This is achieved by maximizing symmetry in the system, and by using different materials with different CTEs to compensate for small asymmetries. Thermal analysis shows that the truss itself does, in fact, contract nearly homologously, and that the overall structure should contract by $2.5 \mathrm{~mm}$ along the length of the boom, with transverse contraction of $0.3 \mathrm{~mm}$ in the transverse direction and $0.3 \mathrm{~mm}$ in the vertical direction. The highest loads are again seen in the flexures that hold the cryostat, but these loads are well within the linear range of the materials. The overall truss design meets all requirements for the scientific objectives.

At present, we have procured all of the components for the carbon fiber truss, and fabrication is under way. We expect to have the complete carbon fiber truss built and instrumented for testing by September 2012. 


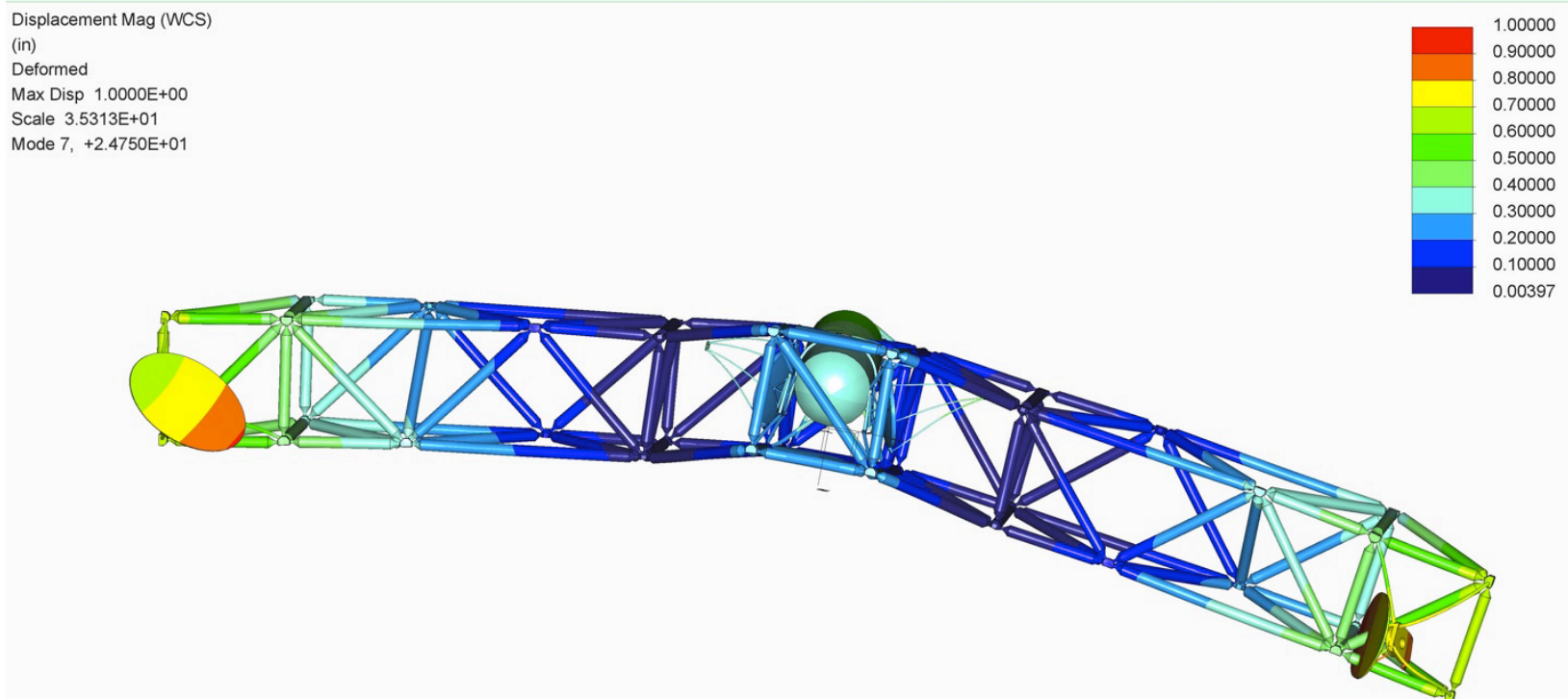

Figure 3: The first mode of vibration of the BETTII metering truss is in the transverse direction, and has a frequency of $24.8 \mathrm{~Hz}$. I.e. the structure is stiff and provides rapid damping of perturbations experienced on flight.

The load frame consists of three boxes constructed of 80/20 aluminum; each is mounted kinematically to the metering truss, with passive vibration damping at the joints. These boxes are designed for holding non-optical system components such as the electronics. The "basement" box, underneath the metering structure, will contain ballast units and the load balancers; the balancing mass on these mechanisms is the batteries for power supply to the payload. This keeps down total weight while providing sufficient mass to keep the gondola in balance during the flight.

\subsection{Cryostat}

The BETTII science instrument resides within a LHe dewar; the mounting of the dewar is particularly important, as it must meet a number of requirements. The dewar mount must:

1) Support the mass of the dewar when subjected to a $10 \mathrm{~g}$ acceleration.

2) Be stiff, to prevent vibration of the instrument.

3) Account for the thermal contraction of the aluminum jacket of the dewar.

4) Maintain a constant position of the dewar windows relative to the beams from the siderostats under temperature variations.

5) Be easily inserted/removed into the metering truss, to enable easy integration and instrument modifications in the field.

Our solution uses four titanium tubes to support the top of the dewar and four titanium tubes to support the bottom of the dewar (Figure 4a). This provides both the strength and stiffness required. The symmetry of the structure guarantees that the dewar will remained centered on the optical path and in the transverse direction as temperature changes. Vertical alignment is maintained by the design of the upper set of titanium tubes; the angle of these tubes compensate for CTE changes in the aluminum of the dewar structure. The bottom tubes are mounted on flexures; this eliminates overconstraint of the dewar, leaving the vertical position of the dewar set entirely by the upper tubes.

Internally, the dewar can be divided into three sections (Figure $4 \mathrm{~b}$ ). The top section is the cryogen space, holding $\sim 7$ liters of LN2 and $\sim 15$ liters of LHe. In addition, this space has a pocket for the He 3 refrigerator needed to provide the $260 \mathrm{mK}$ cold plate for the FIR detectors. While all elements of the cryostat must be rigid and strong enough to withstand a $10 \mathrm{~g}$ acceleration, the mechanical tolerances in this section are easy to achieve..

The center section contains the BETTII science instrument; the FIR channel optics, cold-delay line, and detectors, as described in Section 3. The entirety of this section is made of 6061-T6 aluminum so it shrinks isotropically on cooling. Furthermore, during both testing and flight the entire section (except for detectors) will be held close to $4.2 \mathrm{~K}$ (on-float, a 
manostat maintains constant pressure on the cryogen baths), so ground alignment of the instrument will be maintained on-float. The most difficult element of this design is that the aluminum mirrors must be mounted on rigid structures, as vibration internal to the instrument would greatly reduce the quality of the final science data. This is achieved by using a central optical bench mounted vertically on the cryostat cold plate. This structure provides a very strong and stiff base for mounting of internal optics. Detailed design of the individual optic mounts inside this volume is still underway, but preliminary analysis shows that the required stiffness is easily achieved. The cold plate has a diameter of $400 \mathrm{~mm}$, and the height of this section is $\sim 400 \mathrm{~mm}$.

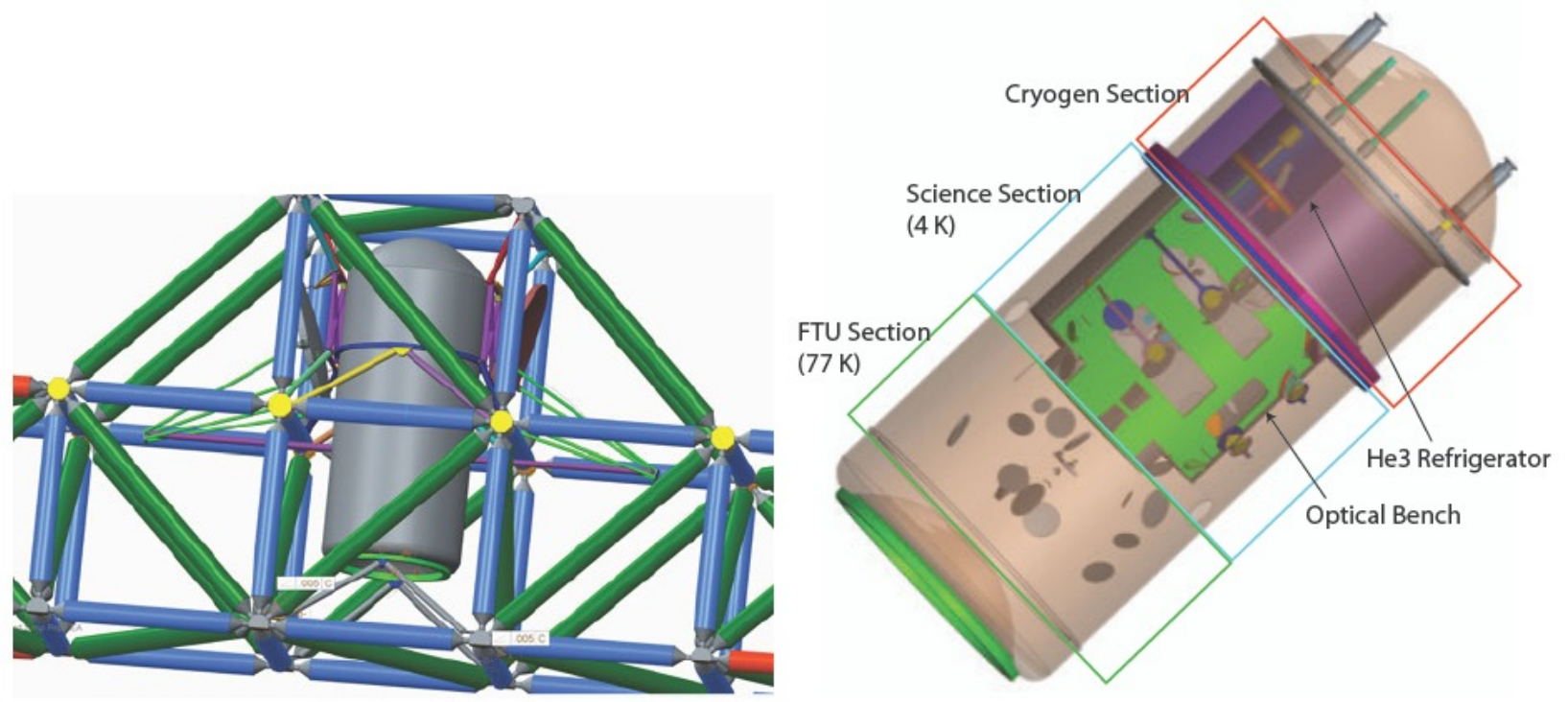

Figure 4: The dewar mounting structure makes use of eight titanium tubes to meet all of the requirements for supporting the cryostat (left). The inside of the dewar makes use of an all-aluminum structure for the optics, insuring consistent behavior as the cryostat is cooled.

The bottom section contains the NIR fringe tracker and the angle sensors. As with the central section, all elements in this fringe tracker are manufactured from 6061-T6 aluminum. These are mounted to a cold plate thermally connected to the LN2 tank, causing the volume to be nearly isothermal at $77 \mathrm{~K}$. The all-aluminum construction has the same alignment advantages as the central section, and because of the more compact design of the optics, meeting the strength and rigidity requirements is somewhat easier (see Rizzo et al. 2012).

The final challenge in the dewar design is to rigidly couple the sections together while maintaining low thermal conductivity between them. This is a common problem for dewars, and the typical solutions of carefully designed stainless steel and G10 connections will be used here. Neither thermal nor mechanical requirements are outside of common practice.

One additional element of the cryostat design is the window itself. Several different window materials were considered, but only polypropylene and diamond crystal provided sufficient transmission in both the NIR (for the fringe tracker) and the FIR (for the science instrument). Because of the extremely high cost of a diamond window, and because a thin polypropylene window has much lower emissivity, the BETTII design uses a polypropylene window, but this creates an additional engineering challenge. Because the window itself is a thin film, it does not have the strength to withstand the pressure differential between the vacuum inside the cryostat and ambient pressure on the ground. This is not an issue at float, because of the reduced atmospheric pressure, but the window must be protected from launch to float. To do this, we use a pair of gate valves (one for each window). These gate valves provide a tight seal over the dewar window while it is pumped down prior to launch. The valves then remain in place until the gondola reaches an altitude of $\sim 110,000$ feet, where the pressure differential is sufficiently low. Once open, the gate valves remain open during operation, and are closed once again prior to gondola drop, in order to protect the dewar window during return to ground. We have already obtained gate valves for testing, and will be able to verify their performance in the near future. 


\subsection{Mechanisms}

In order to achieve the scientific goals of BETTII, a number of mechanisms are required. We have specified requirements for each of these mechanisms, and have been investigating commercial off-the-shelf mechanisms that can meet our criteria. Not included in this discussion is the cold delay line or the gate valves, since those mechanisms are discussed as part of the cryostat section above.

BETTII requires four rotation stages for operation. Two of these stages are used to provide elevation control for the siderostats at the ends of the boom. A third stage controls the rotation of the K-mirror (see discussion in Optical Design, below), and a fourth stage controls the pointing of the star camera. The requirements for these different rotation stages are not uniform, but are sufficiently similar that we have decided to use the same model of device for each of these applications and allows for a single spare for all four applications. This provides a number of advantages in terms of the electrical and software implementation of these mechanisms. During standard operation, each of the four will be commanded to move equal amounts and at equal speeds (although the sign of this motion may be reversed in some cases). However, we will still maintain the ability to control individual stages, in order to compensate for unanticipated deformation of the metering truss or for external perturbations. For our design, we have baselined the Griffin Precision Rotary Table RTS-DD-200; we have one of these devices on hand, and will be testing the device under flight-like conditions over Summer 2012.

As mentioned previously (detail by Rizzo, et al. 2012), the major perturbation of the gondola is due to pendulation. This can lead to large ( $\sim 10 \mathrm{~s}$ of arcminutes) changes in the pointing of the gondola over timescales of up to 20 seconds. To first order, the impact of this motion on the telescope pointing is compensated for using elevation and azimuth control of the gondola itself, but residual motion is removed using a single tip/tilt mechanism in each arm. These mechanisms need to have a large range of travel (up to $\sim 1$ degree) with tip/tilt precision of $\sim 1$ arcsecond. Each of these two mechanisms will operate completely independently, receiving feedback from the Angle Tracker of the Fringe Tracking Unit (FTU). This allows these devices to compensate for both the wide, slow motion of pendulation and for fast shifts due to other perturbations in the system. We have obtained a goniometer and piezoelectric driver that meets our requirement from Newport; however, these devices are not rated to the low temperatures expected at float altitude. We will be testing these devices under flight-like conditions, and have several other off-the-shelf options available (at higher cost) should these devices not function sufficiently well under float conditions.

While the tip/tilt mechanisms can compensate for differential pointing errors in the two arms of the interferometer, the more common problem will be the introduction of a phase difference between the two arms due to mispointing of the entire gondola (due, for instance, to pendulation of the payload). This phase variance will be monitored by the fringe tracker, and will then feed that knowledge to the on-board computers that control the warm delay line. This can then be used to compensate for these slow variations in phase. The warm delay line mechanism needs to have a fairly wide range $(\sim 10 \mathrm{~mm})$ to account for the scale of the pendulation modes, and needs to be accurate to $\sim 100 \mathrm{~nm}$ in order to match phase between the two arms. Our baseline device for this purpose is a Newport stage using a piezoelectric driver identical to that being baselined for the tip/tilt. Testing of the tip/tilt devices will allow us to determine if the piezoelectric driver will work for this application; the stage itself is not expected to encounter problems in the float environment, but should the piezo function sufficiently well, we will procure and test the corresponding Newport stage. Should the piezo device not work, we have identified several other commercial devices that meet or exceed our requirements.

In addition to these mechanisms, we are considering the addition of a tip mechanism for the siderostats. As a result of the optical sensitivity analysis (see below), we determined that one of the tightest tolerances on the optical system is in the co-pointing of the two siderostats. Should the metering truss deform due to thermal effects, the two siderostats can suffer small amounts of differential pointing error, which limits the fringe visibility in the fringe tracker. This problem can be mitigated in several different ways, including a slightly modified optical design and through careful mechanical design. However, we may also include a tip mechanism that provides a few arcminutes of control in the cross-elevation direction (differential pointing errors in the elevation direction can be eliminated through use of the rotation stages). We are still analyzing the requirements for such devices, and determining whether other forms of mitigation can eliminate the need to include them.

For each of the mechanisms discussed here, we have mentioned that the devices will be tested under conditions designed to mirror the on-float environment. To carry out these tests, we have designed and are currently building a Cold Test Chamber (shown in Figure 5). The philosophy of this chamber is to replicate the environmental conditions at float as 
nearly as possible; i.e. a temperature of $\sim-50 \mathrm{C}$ and pressure of $<250 \mathrm{~Pa}$. This pressure can be achieved easily using a Varian IDP-2 pump, and the temperature can be produced using dry ice. In practice, the device to be tested will be mounted to the cold plate, and then the entire chamber will be flipped over. Dry ice will then be placed on the back side of the cold plate, bringing the cold plate to $-80 \mathrm{C}$. The "top" of the chamber is an acrylic bell jar with a 1.5" flat surface on the side; this allows us to pass a laser into and out of the chamber for measurement of the performance of stages. Ports in the aluminum base allow for access for both pump-out and for electrical connections to the mechanisms being tested. Procurement of parts for this chamber is underway, and it should be complete by August 2012; this timing is important to allow full testing of all other mechanisms in the system.

There are three other mechanisms worthy of mention; the Compensated Control Moment Gyro (CCMG), the momentum dump mechanism, and the balancers. The CCMGs are a pair of balanced reaction wheels, mounted on a bearing such that their axes of rotation lie within parallel planes. The wheels, when aligned parallel, are spinning in opposite directions and therefore provide no net angular momentum to the system. By pivoting the two wheels in opposite directions, angular momentum can be inserted into the system, causing the gondola to rotate (changing the azimuth component of pointing). This is a significant component of the BETTII control system, and is discussed in additional detail in Benford et al. 2012.
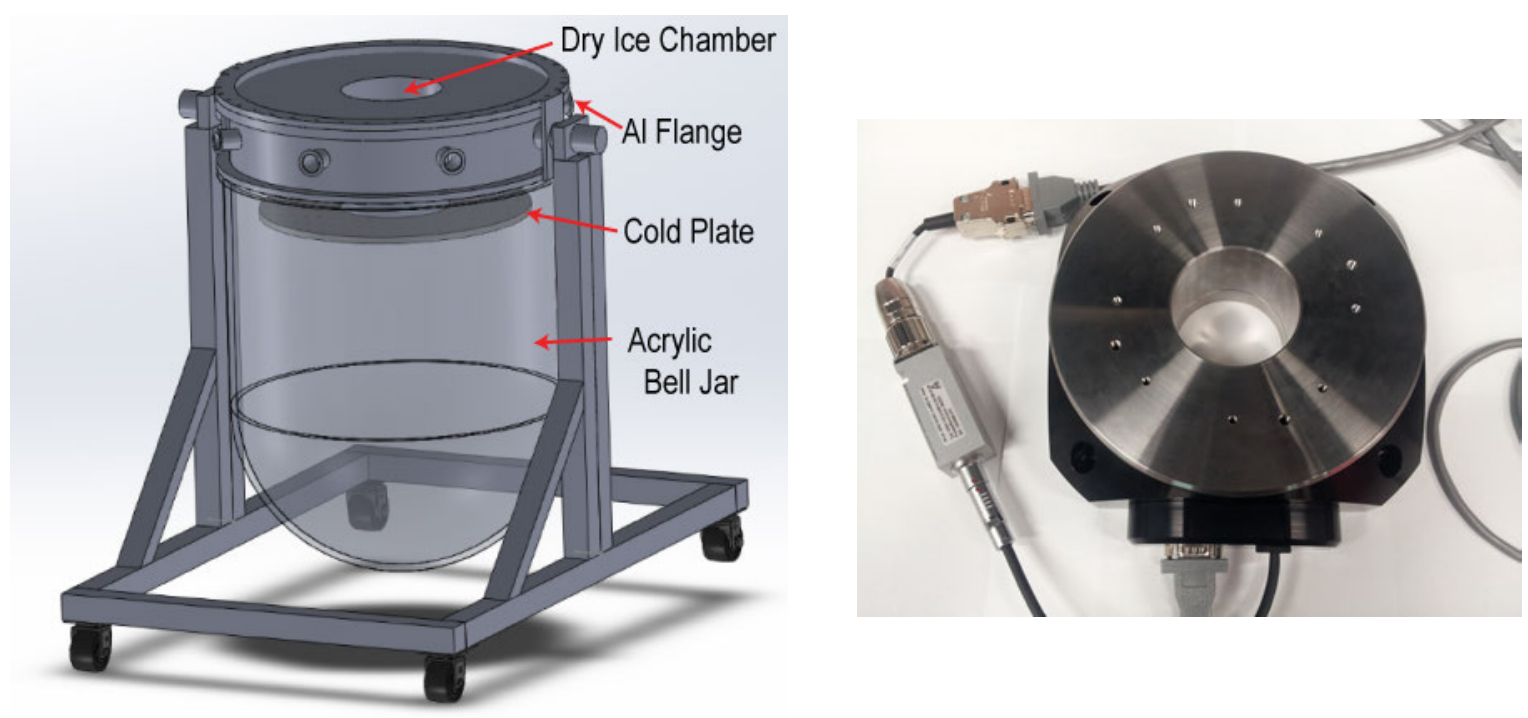

Figure 5: A new environmental test chamber is under construction in the BETTII high bay. This is designed to allow us to operate at low pressure $(<250 \mathrm{~Pa})$ and at low temperature $(\sim 190 \mathrm{~K})$, using dry ice (left). This chamber will be used to test all mechanisms for BETTII, as well as other components for which the on-float environmental conditions might be challenging. The first mechanism to be tested in this chamber is the Griffin rotation stage (right).

The other two mechanisms, the momentum dump and the balancers, are built upon designs that have been previously flown. The momentum dump mechanism is a design like the momentum dump on MSAM (Fixsen et al. 1996). Since BETTII is of similar size and angular inertia, the design and performance will be similar. The balancer is similar to that from ARCADE (Kogut et al. 2004), except that BETTII will use a 2-D system instead of the 1_D system used by ARCADE. In both cases the moved mass is the battery pack. This has the convenience that it is a large mass that is already required, and that it must be removable with only a few connectors.

\section{OPTICAL DESIGN}

The overall optical design is shown in Figure 6. Light is collected by two siderostats at the ends of the metering truss; each siderostat picks up a $50 \mathrm{~cm}$ clear aperture from the sky, and the two mirrors have a center-to-center separation of 8 meters. In each arm, the light travels to a two-element beam-compressing telescope, consisting of a pair of confocal offaxis parabolas. This gives a 20:1 beam compression, with an output of a $2.5 \mathrm{~cm}$ collimated beam. At this point, the two arms differ. In one arm, shown on the left side, the light travels through a four-element warm delay line. The bottom pair of mirrors are mounted on a linear stage, which is moved to compensate for large, low-frequency phase variations between the two arms (as monitored by the fringe tracker). On the other side, the beam passes through a three-element 
K-mirror. As the two siderostats are rotated in elevation, the orientation of their respective fields-of-view rotate, but in opposite directions. The K-mirror serves as a field rotator. By slaving the rotation stage upon which the K-mirror is mounted to the siderostats elevation pointing, the K-mirror counterrotates the beam from the right side of the interferometer such that the field rotation matches exactly that of the left arm of the interferometer. Both beams then enter the cryostat through polypropylene windows.

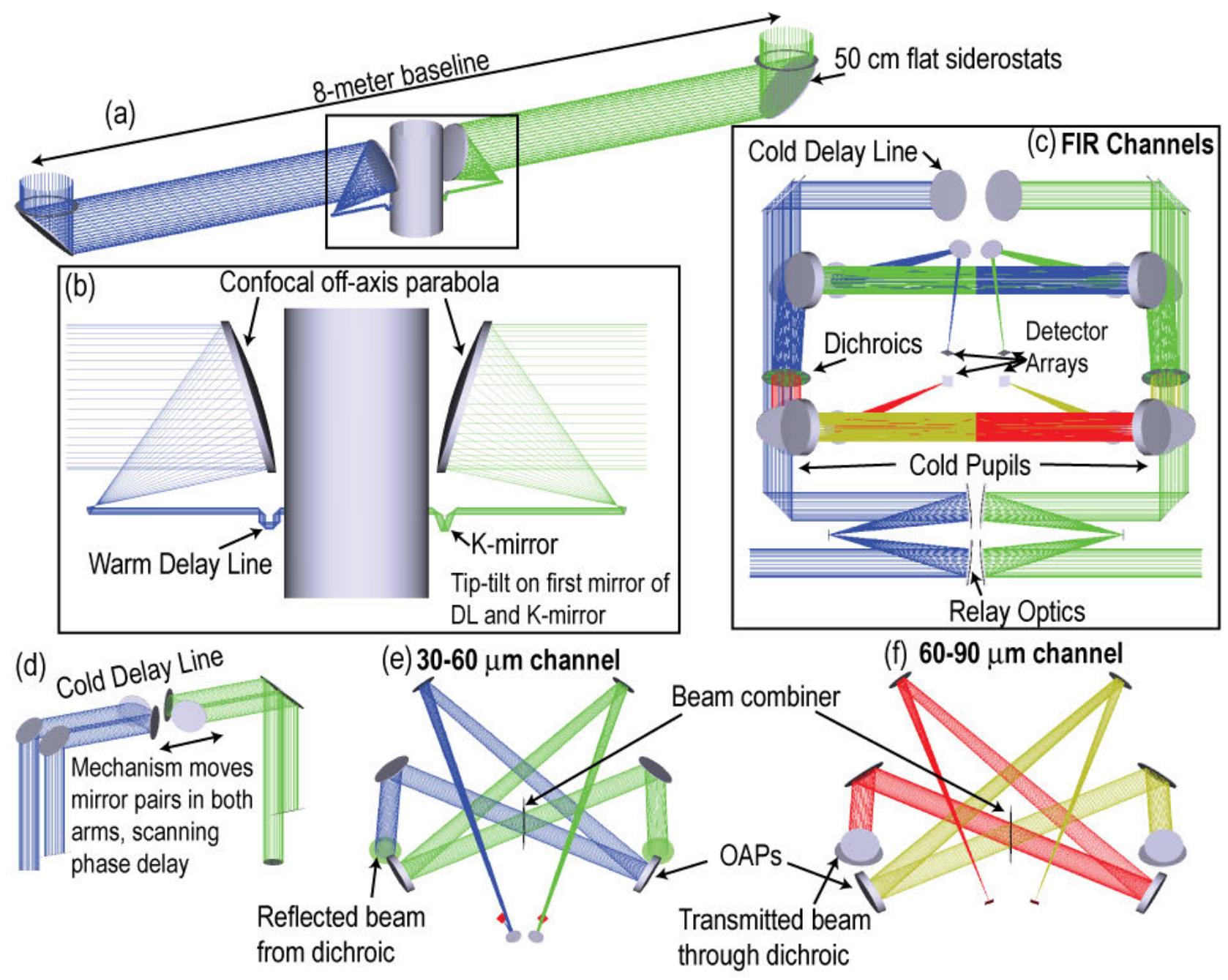

Figure 6: BETTII collects light on an 8-meter baseline with a pair of siderostats (a), which steer the beams to confocal telescopes (b). In the left arm, the collimated beam then passes through a warm delay line; in the right arm, it passes through a K-mirror. The two beams then enter the cryostat (c). A dichroic just inside the dewar (not shown) reflects the NIR light used for fringe tracking, while the FIR beams pass through relay optics then through a cold stop. The beams then travel through flat mirrors to the cold delay line (d). The beams then travel back down to dichroics in each arm, which reflects the short wavelength channel $(30-50 \mu \mathrm{m})$ and passes the long wavelengths $(60-90 \mu \mathrm{m})$. In the short wavelength channel (e), the light passes through another flat, then through the beam combiner. The beams are then focused onto the detectors, through an additional fold mirror because of the long focal length of the camera. The longwavelength channel (f) is similar, but has a shorter focal length such that it does not require the additional fold mirror.

Inside the cryostat, the collimated beams pass through a dichroic that reflects the NIR portion of the beam (this is not shown in Figure 6). The NIR beam then passes to the FTU, as discussed in detail by Rizzo et al. 2012. The FIR beams pass through this dichroic then through a set of relay optics; each of the two relays include a pair of off-axis parabola (OAPs) and a single field mirror. This allows for accurate placement of the beam onto the cold pupil. Following the pupil, the FIR beams travel to a flat mirror that guides them into the cold delay line. The cold delay line consists of a 
pair of back-to-back rooftop mirrors mounted on a single stage; as the stage moves, it increases the path length in one arm while decreasing it in the other, providing the interferometric phase modulation with a $\mathrm{x} 4$ lever effect. Following the delay line, each beam passes back through a flat mirror to a dichroic that splits it into two bands. The short band, reflected off the dichroic, covers approximately $30-50 \mu \mathrm{m}$, while the long band covers 60-90 $\mu \mathrm{m}$ (the exact break point between the bands will depend upon the architecture of the dichroics, but we expect for it to fall in the 50-60 $\mu \mathrm{m}$ range). In the short band, the light in each arm reflects off of a single flat mirror and then the two beams meet at the beam combiner, producing two interfered output beams. These beams continue to OAPs that focus them on the FIR arrays via a pair of flat mirrors (needed because of the long focal length in this channel). The long band is similar, using the beams transmitted by the dichroics.

We have analyzed the optical design to study the potential impact of differential wavefront error and beam shear to specific perturbation tolerances and optical alignment requirements. For this purpose, the FIR science channels do not drive the constraints on alignment and tolerances; the NIR fringe tracker is the limiting system. From our sensitivity analysis, we have found that the following three elements are the most critical from a design perspective:

1) Pointing errors resulting from differential motion of the siderostat mirrors or of the afocal telescope systems. The images from the two telescopes are made coincident at the detector by compensation with the tip-tilt mechanisms. However, this compensation occurs after the beams have passed through the telescopes, generating differential wavefront error. This is a field-dependent effect, and by restricting the source position of the star being used for fringe tracking to within 6 arcseconds of the boresite, this sensitivity is greatly reduced. We are considering other methods for mitigation (such as tip control of the siderostats), as that would provide additional flexibility for choice of tracking star.

2) These same pointing errors also generate beam shear at the beam combining optic within the fringe tracker. Unlike the wavefront error, this is field independent, so mitigation by restriction of the tracking star location does not work. However, by adjusting the optical design such that a pupil image is formed on the tip-tilt mirrors dramatically reduces the shear, effectively eliminating this problem. This does not, however, mitigate the wavefront error mentioned above. Our current optical design is a two-mirror system; we are redesigning this portion of the optics as a three-mirror afocal relay to place a real pupil on the tip-tilts. The beam is still collimated going into the cryostat, so this has no impact on other portions of the optical path.

3) Differential motion of the telescope secondary mirror(s) relative to the primary mirror(s) also generates wavefront error. While we are able to align the two mirrors to the required tolerance on the ground, variations in the structure due to temperature variations can lead to differential motion of these mirrors. We are mitigating this somewhat through careful mechanical design of the mirror mount structure, but this is not entirely sufficient because when the experiment is at float altitude, the structure itself will not be isothermal. We are continuing to explore this mechanical design, and are considering the inclusion of a heat pipe system to try to make the structure isothermal during flight.

While not strictly part of the optical design of the experiment, we include here a brief discussion of metrology systems. The FTU will provide us a direct measurement of differential piston errors occurring between the two arms. The Angle Tracker component of the FTU will measure the pointing changes in each of the two arms; however, such changes can be caused by either motion of the siderostat or by changes in the pointing of the telescope assembly. In order to differentiate between these two possibilities, we are planning on using a diode laser and a quad cell. Light from the laser will be reflected from a mirror mounted at the end of the boom, and will return to the quad cell. This will allow us to measure the small deflections in the boom that are responsible for changes in the pointing of the siderostats, breaking the degeneracy between the two possible sources of pointing error.

\section{DETECTORS}

BETTII's optical layout feeds a set of four nearly identical detector arrays, with a nominal 2' field of view for each. The short wavelength band, covering $30-50 \mu \mathrm{m}$ (at a minimum), is illuminated such that a point source produces a white light image with a size of roughly $19^{\prime \prime}$ in diameter; the long wavelength band, which reaches out to $90 \mu \mathrm{m}$, would have a spot size of approximately $34^{\prime \prime}$ diameter. In order to enforce as much commonality between the detector arrays as possible, the format (both in pixel count and pitch) will be the same; hence, the illumination of the detectors varies. BETTII will use a $9 \times 9$ array, which for the shorter band is illuminated with a plate scale of 13.3 "/pixel for a full $2^{\prime}$ field of view; at 
the longer band, the plate scale is set to $16.7^{\prime \prime} /$ pixel for a $2.5^{\prime}$ field of view. The oversampling of the point spread function in the longer band is therefore 1.4 times the oversampling in the shorter band.

For these wavelengths, the highest performance detectors are superconducting transition edge sensor bolometers (Allen et al. 2006) read out by SQUID multiplexers (Irwin et al. 2004). These have been demonstrated to have excellent performance (Benford et al. 2000) and low noise (Staguhn et al. 2004), and are commonly in use in multiple groundbased instruments (e.g., Staguhn et al. 2009) and are under development for balloon-borne astrophysics (e.g. Benford et al. 2010). The BETTII bolometer arrays will have 81 optically active pixels, 2 dark pixels, and 1 frame-mounted detector that are read out by a $4 \times 22$ SQUID multiplexer. The 4 spare channels are left disconnected to monitor any signals in the amplifier and electronics chain that do not arise from detectors. These SQUIDs feature flux-switched time domain multiplexing for very low power operation and have been designed with high-order gradiometric coils to reduce pickup from stray magnetic fields.

At balloon altitudes, the residual atmospheric emissivity is very low (Harries 1980), and so the dominant power loading the detectors is from the mirrors and cryostat windows. The BETTII bolometers have a saturation power designed significantly above this to accommodate suboptimal mirror emissivity, and yet the expected detector noise equivalent power (NEP) is still well below that of the incident photon stream (Table 1). In order to respond promptly to interferometric power modulation, the detectors are required to have time constants of less than $0.6 \mathrm{~ms}$; we predict that this requirement shall be exceeded by a factor of 2-3. This can all be achieved at a transition temperature of $450 \mathrm{mK}$, well within reach of readily available ${ }^{3} \mathrm{He}$ sorption coolers.

Table 1. BETTII Detector array parameters

\begin{tabular}{|l|c|c|l|}
\hline Parameter & SW Band & LW Band & Units \\
\hline Wavelength & 38 & 67 & $\mu \mathrm{m}$ \\
\hline Bandwidth & $51 \%$ & $60 \%$ & \\
\hline Atmospheric Power & 35 & 36 & $\mathrm{pW}$ \\
\hline Optics Power & 148 & 56 & $\mathrm{pW}$ \\
\hline Saturation Power & 550 & 295 & $\mathrm{pW}$ \\
\hline Photon NEP & $1.4 \cdot 10^{-15}$ & $7.4 \cdot 10^{-16}$ & $\mathrm{~W} / \sqrt{\mathrm{Hz}}$ \\
\hline Detector NEP & $2.2 \cdot 10^{-16}$ & $1.6 \cdot 10^{-16}$ & $\mathrm{~W} / \sqrt{\mathrm{Hz}}$ \\
\hline Time Constant & 0.2 & 0.3 & $\mathrm{~ms}$ \\
\hline Transition Temp. & 450 & 450 & $\mathrm{mK}$ \\
\hline Format & $9 \times 9$ & $9 \times 9$ & pixels \\
\hline Pixel Scale & 13.3 & 16.7 & arcsec \\
\hline
\end{tabular}

\section{ELECTRICAL}

The preliminary BETTII electrical block diagram is shown in Figure 7. The flight computer ("Ford") interprets commands as received from the Consolidated Instrument Package (CIP; provided by the Columbia Scientific Ballooning Facility), collects science detector data from the Multichannel Electronics (MCE), and communicates with other computers via Ethernet. The Synchronization Box (Sync Box) provides the master clock to synchronize system timing across the instrument. The Compact Real-Time Input-Output (cRIO, "Boop") consists of a primary 9802 controller along with three 9144 expansion chassis and provides many of the component interfaces, mechanism control, and redistributes clocks. External/interstitial sensor/actuator electronics were minimized in favor of cRIO modules in order to simplify the design, reduce interfaces, and reduce extraneous power conversion and clocking; such cRIO systems have been demonstrated at aircraft altitudes. The Star Tracker/Fringe Tracker computer ("Grable") collects star tracker images, autofocuses the star camera, collects H1RG detector information from the Leach electronics, and provides fringe tracker error signals to the cRIO. Modifications may be required to adapt COTS components to the BETTII architecture and environment. Several components come with integrated DC-DC converters, others will be provided with the required regulated voltage from custom built regulator circuits. Thermal management may implement a pressurized electronics enclosure to allow forced-air convective cooling, thermal strapping to increase conduction from warm components, or a combination of both.

The power system baseline is standard CSBF issue lithium sulphur dioxide, $3 \mathrm{~V}$ batteries in packs of 10-12. Since some elements require linear regulated voltages, some battery packs will be tailored to significantly increase efficiency and reduce battery requirements. There will be a separate set of voltage regulation, switching, and distribution electronics. Separate batteries will also be used to provide isolation of particularly noisy or sensitive components. The preliminary average power estimate is $\sim 1 \mathrm{~kW}$. Since battery capacity at $-40 \mathrm{C}$ is about $30 \%$ of that at room temperature, keeping batteries warm significantly reduces weight. Previous experience has demonstrated that self-heating in an insulated housing can keep batteries warm on float. Other heat dissipating components may be included with the batteries to provide additional heat. Connector panels will also be included to allow for modular development and testing of subsystems as well as ease instrument disassembly for shipping and subsequent reintegration. 


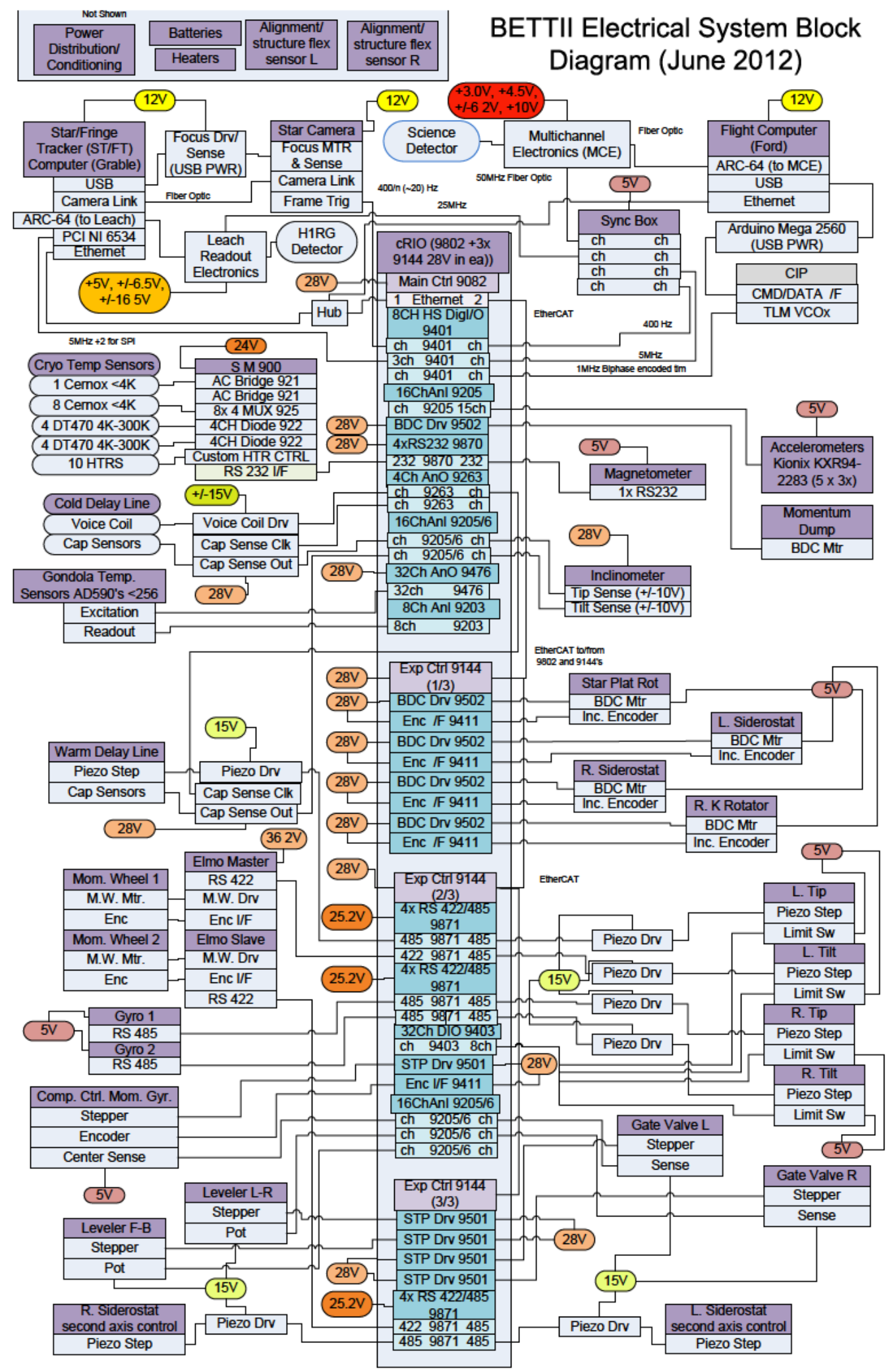

Figure 7: The BETTII electrical system includes a large number of components; the current block diagram for the electronics is shown here. 
Since the BETTII structure is non-conductive, copper braid will be used to provide low-impedance grounding of electrical components. Harnesses will tie internal shields on the source end and the overbraid on both ends to extend the chassis/Faraday cage between boxes.

\section{THE PATH FORWARD}

At present, we are planning BETTII for its first launch in Spring 2015, followed by analysis of science data and the performance of the gondola and instrument. In order to meet the launch goal, and to provide the highest probability of scientific success, we have generated a detailed design that encompasses all major components of the balloon. Over the coming months, we will continue our design effort, focusing on the more minor subsystems that are needed for flight. In parallel, we are now beginning the fabrication and testing efforts.

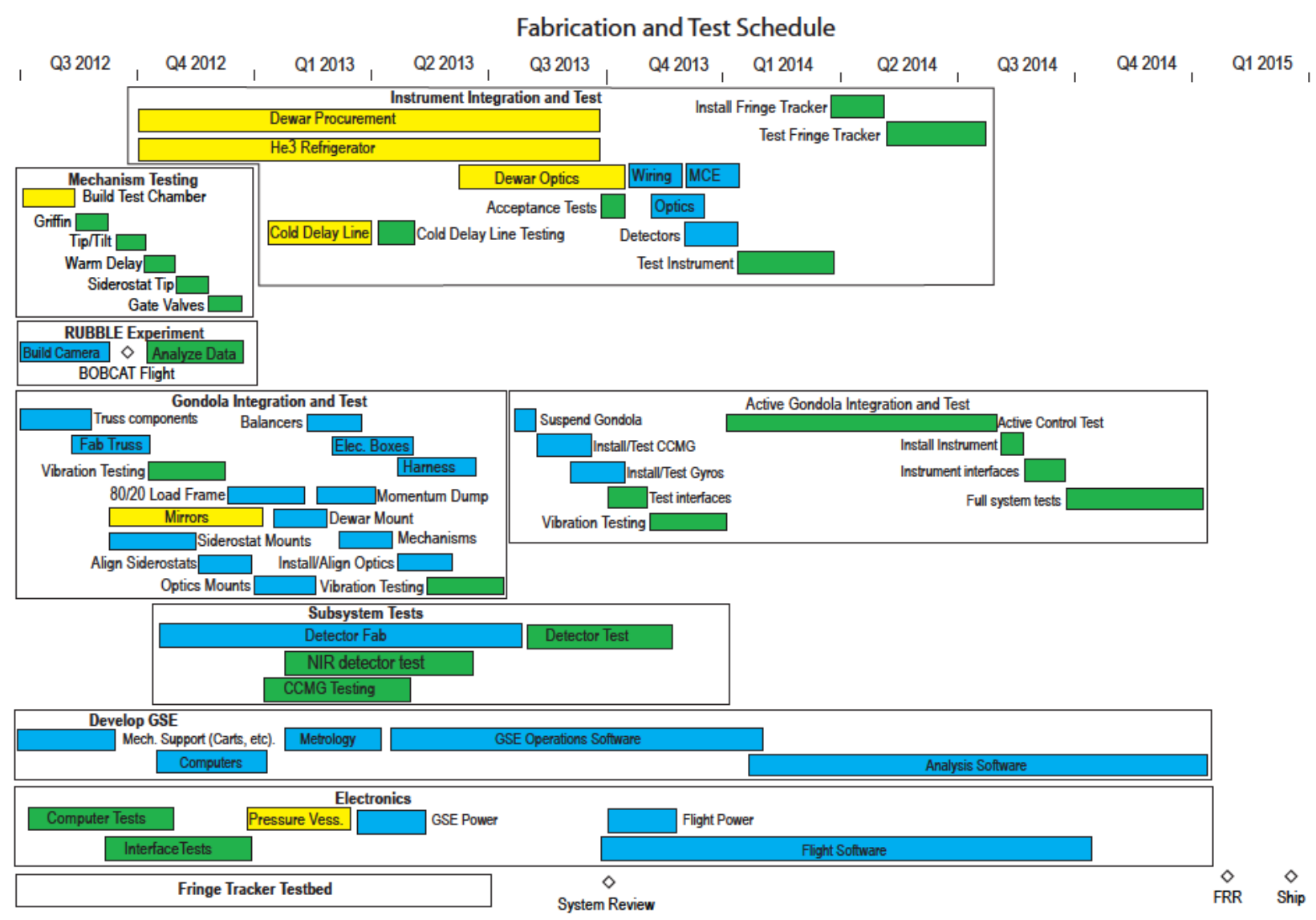

Figure 9: The schedule for fabrication and test of BETTII leads to a launch in spring 2015.

\subsection{Near-term (through end 2012)}

Up to this point, the effort on BETTII has been focused on design of individual elements and upon the system engineering of the payload. We have now begun the procurement/testing phase, having obtained all of the components needed to assemble the metering truss and mechanisms for testing. At this time, we are laying up the individual truss components by gluing the nosecones into the carbon fiber tubes. Following curing, each of these tubes is being tested to verify that the bond meets strength requirements; based upon previous tests and with some minor process improvements, we do not expect any failures, but want to be ensure that any improperly bonded truss components are identified and fixed. As the truss components are completed, they will be painted white for flight, and then we will start to assemble the metering truss. We expect that the metering truss will be complete by the end of September 2012. In Fall 2012, we will attach accelerometers to the structure and will test the vibration modes of the gondola to confirm that the structure conforms to our structural models. 
Following vibration testing, we will assemble the siderostat mount structure and will begin procurement of the large mirrors needed for BETTII. We expect that the siderostat mirrors, which are simply lightweight aluminum flat mirrors, shall not require a long lead time, and we anticipate being able to align the two siderostats to the long axis of the gondola by the end of 2012 .

We are also developing the mechanical Ground Support Equipment (GSE) for BETTII. BETTII is being constructed within the Building 20 High Bay at NASA Goddard. To support the assembly, we are building a simple cart for moving the completed structure, and are determining if an A-frame is desirable for suspension of the gondola (without requiring use of the lifting crane). We are also beginning to develop the ground-system more generally, including the electronics and the procurement of metrology systems needed to test and align the structure and the mounted optics.

Simultaneously, we are currently building up the environmental test chamber. This will be a valuable addition to our suite of tools, as it will be used to test a wide range of components of BETTII. The first series of tests will be for the mechanisms. The individual devices are being tested in the lab now under ambient conditions, and when the chamber is complete we will test under environmental conditions similar to float. We expect these tests to continue through the Fall 2012, and by the end of the year, we will have validated the mechanisms used within BETTII. Should one of these mechanisms be shown to not work, we will procure alternate off-the-shelf devices for test.

We have already procured a number of our electrical components; in particular, the cRIO module, the FT computer, and the NIR detector readout electronics (Leach boards). These components are currently in test, and we are beginning design of the software interfaces between these and other devices.

We are also currently working on developing the RUBBLE experiment to fly in September 2012. This is discussed in additional detail in Benford, et al. 2012. This experiment will "piggy-back" on the Bobcat balloon payload, and we validate our star camera design (and associated sensors and hardware) for BETTII. As part of this effort, we are developing the first segment of flight software needed for BETTII.

\subsection{Mid-Term (2013)}

Following the alignment of the siderostats, we will assemble and attach the telescope assembly, the dewar mount, and the load frame structure. The telescope assembly will be fabricated in two steps; the structure itself will be assembled independently of the gondola, and the mirrors will be attached to this structure. We will then test this structure independently to verify that the alignment of the telescope optics meet requirements. The telescope assembly structure will be mounted to the metering truss, and will then be aligned to the siderostats. Assembly and integration of the dewar mount and load frame structure will happen in parallel.

With the telescope assembly in place, we will attach the external mechanisms to the metering truss. Following completion of the mechanism tests, we will procure any additional mechanisms required (e.g. the additional 3 rotation stages), and each of these will also be validated in our environmental test chamber. These will then be ready for inclusion in the gondola structure (this will actually occur within 2012, so that we have the rotation stages available for mounting the siderostats). The first step will be assembly of the optics structure on each stage; the tip-tilt and fixed optics will be mounted on a rotation stage for the K-mirror assembly. The tip-tilt and linear motion stage will be mounted on a single plate as the warm delay line. We will align the optics on each of these assemblies prior to mounting to the gondola, and then they can be mounted to the gondola structure. Once mounted, we will verify the alignment of all the external optics, ensuring that the beams from both arms of the interferometer are collinear. We are presently defining an alignment program, following the philosophy described here; we will align each optical element as it is incorporated into the gondola structure.

By mid-2013, the entire gondola structure, including optics, will be assembled, and we will conduct additional vibration tests, to again verify that the models accurately represent the structure. These tests will also include operating each of the mechanisms individually, to determine if these mechanisms cause any vibrations in the metering structure and if so to characterize this behavior. The gondola will then be ready for suspension within the laboratory, and we will move on to installation of the control system. This will include installation and test of the compensated control moment gyros (CCMG), the rate-sensing gyros, and the associated electronics. We will the conduct additional tests to measure any vibration induced by the CCMG in the structure.

Procurement, fabrication, and assembly of the instrument itself will occur through all of 2013. The cryostat and the He3 refrigerator are both expected to be long-lead items, taking as long as 9 months for acquisition. We plan on issuing orders for both of these components at the beginning of 2013, although we are working to see if we can get these orders 
in place as early as August 2012. When the cryostat arrives, we will install thermometry and will then perform acceptance tests on the dewar, allowing us to measure parasitic heat loss (hold time), etc. We will conduct acceptance tests for the He3 refrigerator as well.

Once the procurement for the cryostat has been started, we will assemble the internal optical elements for the instrument. We will fabricate the optical bench and will mount and align the optics to this plate. Once these are mounted, we will align all of the optics on the optical bench. Simultaneously, we will build the cold delay line, and we will test at LHe temperatures to validate performance prior to the cryostat arrival. Once the cryostat has been accepted, we will begin installing the instrument itself. This includes the electrical wiring for the detectors, the He3 refrigerator, the cold delay line, the detectors, and then the optics. This should be a rapid process, and we anticipate having all of these elements installed by the end of 2013 .

Simultaneously, we can assemble the FTU. While we have not discussed this design in detail, one of the key elements of the design is that the FTU sits entirely within the LN2 volume. One of the advantages of this is that the entire FTU assembly can be built in parallel to the FIR instrument, and can then be mounted in place within the instrument. Assembly and integration of the fringe tracker into the instrument is not planned to occur until 2014.

Fabrication of the FIR detector arrays will begin in Fall 2012, and we anticipate having the complete detectors and associated electronics (both cold and warm) in hand by early 2013. Testing and calibration of these detectors will occur during 2013, and we will be integrating the detectors into the cryostat by the end of the year. We are also obtaining H1RG NIR detectors for the FTU. We expect to have these on hand in early 2013, and we will test and characterize these detectors before their inclusion in the FTU.

Finally, during this year, we will build on the software developed for RUBBLE to build the full flight software package. Much of the design work for this software has already been completed, but as the electronics and mechanisms are completed, we will continue to refine this design to reflect the actual flight hardware. Our overall design will make use of modular code development, allowing us to test modules independently as individual components become available. Generally, software development will occur in parallel to hardware development.

\subsection{Long-Term (2014-2015)}

By the beginning of 2014 , both the instrument and the full gondola structure will be complete. At this time, we will begin major system-level tests. For the gondola, this will consist largely of testing the ability to control motion of the structure. This is a critical step, as it will demonstrate that we are able to point the gondola with the required level of precision and maintain that pointing accurately, without inducing significant vibration in the structure.

At the same time, we will be testing the instrument itself, using a FIR light source to verify the alignment of the system and the operation of the cold delay line. We are also exploring the feasibility of developing a coherent light source that could be used to measure FIR fringes within the instrument. Results from these tests will feed directly into the ongoing software development for data reduction.

At the beginning of 2015, we will have our Flight Readiness Review (FRR), discussing results from all tests and showing that we are ready for flight. Following the FRR, we will have 1-2 months to address any issues that might arise within the review, and then we will be ready to ship the payload to the launch site in time for a spring 2015 launch.

Following launch, assuming a successful mission operation, we will analyze both the science data from the flight and the flight telemetry data itself. The telemetry data will allow us to characterize the behavior of the payload during flight. This information will be valuable for the design of future balloon-borne interferometry experiments, and for other payloads that have tight pointing requirements.

\section{BEYOND BETTII}

While BETTII is primarily motivated by potential for important scientific discovery, it has the additional benefit that it paves the way for future space-based interferometers. In the near term, a space-based interferometry mission such as SPIRIT or FIRI would provide ground-breaking new scientific data in the far-infrared, and would lead to a much improved understanding of star and planet formation, galaxy evolution, and the enrichment of the interstellar medium (Leisawitz \& Rinehart 2012). In the longer-term, longer baselines in the infrared will provide the angular resolution to match the capabilities of Hubble and JWST, while at shorter wavelengths interferometers such as Planet Imager will provide the first capability to produce detailed images of exoplanets. While such ambitious missions may be decades 
away, BETTII will provide the first demonstration of this capability, and will be a critical step towards bringing such missions to fruition.

\section{REFERENCES}

[1] Allen, C. A., Benford, D. J., Chervenak, J. A., Chuss, D. T., Miller, T. M., Moseley, S. H., Staguhn, J. G., and Wollack, E. J., "Backshort-Under-Grid arrays for infrared astronomy," Nuclear Instruments and Methods in Physics Research A 559, pp. 522-524 (2006)

[2] Benford, D. J., et al. "Multiplexed readout of superconducting bolometers," International Journal of Infrared and Millimeter Waves 21, 1909-1916 (2000)

[3] Benford, D. J., et al. "5,120 superconducting bolometers for the PIPER balloon-borne CMB polarization experiment," Proc. SPIE 7741, 77411Q (2010)

[4] Benford, D. J., et al., "Precision attitude control for the BETTII balloon-borne interferometer," Proc. SPIE 8444, 8444-97 (2012).

[5] Fixsen, D. J., Cheng, E.S., Cottingham, D.A., Folz, W.C., Inman, C.A., Kowitt, M.S., Meyer, S.S., Page, L.A., Puchalla, J.L., Ruhl, J.E., \& Silverberg, R.F. "A Balloon-borne Millimeter-Wave Telescope for Cosmic Microwave Background Anisotropy Measurement," ApJ 470, 63 (1996).

[6] Harries, J. E. "Atmospheric radiometry at submillimeter wavelengths," Applied Optics 19, pp. 3075-3081 (1980)

[7] Irwin, K. D. "SQUID multiplexers for transition-edge sensors," Physica C Superconductivity 368, pp. 203-210 (2002)

[8] Kogut, A., Fixsen, D. J., Levin, S., Limon, M., Lubin, P. M., Mirel, P., Seiffert, M., \& Wollack, E. “An Instrument to Measure the Temperature of the Cosmic Microwave Background Radiation at Centimeter Wavelengths," ApJS 154, 493 (2004).

[9] Leisawitz, D.T. \& Rinehart, S.A. "The path to far-IR interferometry in space: recent developments, plans, and prospects," Proc. SPIE 8442, 8442-73 (2012).

[10] Rinehart, S. A. "The Balloon Experimental Twin Telescope for Infrared Interferometry (BETTII)," Proc. SPIE 7734, 16 (2010).

[11] Rizzo, M. et al. "Tracking near-infrared fringes on BETTII: a balloon-borne, 8m-baseline interferometer," Proc. SPIE 8445, 8445-63 (2012).

[12] Staguhn, J. G., Moseley, S. H., Benford, D. J., Allen, C. A., Chervenak, J. A., Stevenson, T. R., \& Hsieh, W.-T. "Approaching the fundamental noise limit in Mo/Au TES bolometers with transverse normal metal bars," Nuclear Instruments and Methods in Physics Research A 520, 336-339 (2004)

[13] Staguhn, J., et al. "New Results from GISMO, a 2 mm Camera Using a Backshort-Under-Grid TES Bolometer Array," Submillimeter Astrophysics and Technology: a Symposium Honoring Thomas G. Phillips 417, 451 (2009)

\section{ACKNOWLEDGEMENTS}

The author would like to thank all of the members of the BETTII team for their contributions. Much of the work carried out on the BETTII project has been completed with the assistance of undergraduate student interns at Goddard Space Flight Center. Many thanks to all of these students, including: S. Gomillion, B. Hoffman, W. Tierney, C. Wagner, A. Cotto, R. Curley, T. Kale, T. Handleton, Y. Okafor, N. Mihalko, P. Nehme, L. Oliviera, A. Gore, H. Spooner, and J. Doiron. This work is being carried out under a grant issued through the NASA ROSES/APRA program. The author also wishes to thank the Astrophysics Science Division at Goddard for supporting the development of the BETTII proposal. 